\title{
PRESENCIA DE LAS MUJERES EN LA ALTA GESTIÓN UNIVERSITARIA. LAS UNIVERSIDADES PÚBLICAS EN CHILE
}

Presence of women in university management. Public universities in Chile

\author{
SARA DEL PINO ARRIAGADA ${ }^{1}$ \\ ROSANA VALLEJOS CARTES ${ }^{2}$ \\ LUIS AMÉSTICA-RIVAS ${ }^{3}$ \\ EDINSON CORNEJO-SAAVEDRA ${ }^{4}$ \\ Universidad del Bío Bío. Chile. Correspondencia: \\ sdelpino@ubiobio.cl, rosana.vallejos@gmail.com, \\ lamestica@ubiobio.cl, ecornejo@ubiobio.cl
}

Recibido: 23-03-2017
Revisado: 15-08-2017

Aceptado: 16-08-2017

Resumen: Este estudio se propone revisar la participación de las mujeres en la gestión de las universidades públicas chilenas durante el año 2014 y analizar techos de cristal que impiden su ascenso. De 847 cargos directivos, 206 son ocupados por mujeres $(24,3 \%)$ : esta participación se concentra en decanaturas o direcciones de departamento, y resulta baja en los niveles superiores. A través de un modelo logit, se mide su desempeño mediante variables de años de acreditación, presencia en ranking QS e indicadores financieros. Se establece que no hay significancia estadística que permita inferir que el desempeño de las universidades depende de la presencia de mujeres u hombres en la gestión.

Palabras clave: mujeres; gestión universitaria; educación superior; techo de cristal; desempeño.

Abstract: This study aims to review the participation of women in the management of Chilean public universities during 2014 and to analyze glass ceilings that prevent their promotion. Out of 847 management positions, 206 are held by women (24.3\%): this participation is concentrated in deaneries or department directors, and is low at higher levels. Through a logit model, its performance is measured considering variables like years of accreditation, presence in QS ranking and financial indicators. It is established that there is no statistical significance to infer that the performance of universities depends on the presence of women or men in management.

Keywords: women; university management; higher education; glass ceiling; performance.

\footnotetext{
${ }^{1}$ Magíster en Dirección de Empresas, egresada del Programa MDE, Facultad de Ciencias Empresariales, Universidad del Bío Bío (Chile).

2 Magíster en Planificación y Desarrollo Rural, Universidad de Guelph (Canadá). Investigadora del Centro de Agronegocios, Facultad de Ciencias Empresariales, Universidad del Bío Bío (Chile).

${ }^{3}$ Doctor en Administración y Dirección de Empresas, Universitat de Catalunya (España). Académico del Departamento de Gestión Empresarial, Facultad de Ciencias Empresariales, Universidad del Bío Bío (Chile). Investigador asociado grupo de investigación en Direcció Universitaria de la Universitat Politécnica de Catalunya (España).

${ }^{4}$ Master in Business Administration, ILADES-Georgetown University (Estados Unidos). Académico del Departamento de Gestión Empresarial, Facultad de Ciencias Empresariales, Universidad del Bío Bío (Chile).
} 


\section{INTRODUCCIÓN}

La participación de la mujer en la educación superior en los últimos años ha alcanzado niveles mayores a la de los hombres, experimentando importantes cambios en el ámbito del trabajo que se reflejan en mayores niveles de calificación, de participación en el mercado laboral y de experiencia profesional (Guil, 2014; Lorenzo, Sola y Cáceres, 2007). No obstante, en el plano de su ocupación en cargos gerenciales, tanto a nivel internacional como en Chile, la situación es distinta y comparable con la baja participación de las mujeres en política, ejemplo de ello es el estudio de Barrientos y Hess (2016) donde se muestra que la participación femenina en los directorios y plantas de alta gerencia de 85 empresas chilenas que cotizan en bolsa llega a un 4,5\% en el año 2015. En este sentido a nivel internacional, la Comisión Europea en 2011 hizo un llamado a las empresas a promover la incorporación de mujeres en los órganos de decisión hasta alcanzar en 2015 un 30\%de participación en los consejos de administración y un 40\% en el 2020 (Larrieta, Velasco y Fernández, 2015). En el caso del sector público chileno, aunque se observan avances en el ámbito del Estado con mayores tasas de participación de mujeres en cargos directivos, éstas aún siguen siendo bajas, situación también observable en el sector privado, donde diversos estudios indican que esta es una situación estructural de la sociedad chilena en su conjunto (Benvin y Perticara, 2007; Brega, Durán, y Sáez, 2015; Cárdenas, 2013; Meller, Valdés, \& Lara, 2011).

Los diagnósticos sobre la participación de la mujer en la educación superior evidencian avances en el mejoramiento de la situación de desigualdad de género, no obstante, persisten dificultades para que las mujeres accedan a puestos estratégicos en la toma de decisiones y en los puestos de poder (Kiss, Barrios y Alvarez, 2007; Ordorika, 2015; Sugur y Cangöz, 2016). La experiencia internacional indica al año 2010 que el 10\% de los rectores son mujeres en veintisiete países de la Unión Europea, siendo Suecia el de mayor presencia con un 43\% (Peterson, 2016). Por ello, al igual que lo sucedido en otros países, en Chile una primera aproximación a la cantidad de académicas involucradas en educación superior en el año 2014 indica que ellas representan el 42,7\% del total de académicos, en tanto en las universidades públicas las mujeres son un $36,4 \%$ del total (Ministerio de Educación de Chile, 2014). Lo anterior está en sintonía con el crecimiento explosivo que ha tenido el sistema y refleja que las instituciones operan en un ámbito altamente competitivo, donde existe una fuerte disputa por los alumnos, por fondos de investigación y por captar el mejor personal académico (Améstica, Gaete y Llinàs-Audet, 2014).

En Chile, Berríos (2007) pudo evidenciar que un $23 \%$ de los cargos directivos superiores en universidades chilenas eran ejercidos por mujeres y que solo el $3 \%$ ejercía el cargo de rectora. En el año 2013 en las universidades chilenas solo el 5,1\% son rectoras, mientras el $22,6 \%$ son decanas o jefas de carrera. De los 39 Institutos Profesionales considerados, en $25,6 \%$ son rectoras, mientras en $32,7 \%$ son jefas de carreras (Cárdenas, 2013). Según los datos extraídos del Consorcio de Universidades Estatales, las 16 universidades son lideradas por rectores hombres (Consorcio de las 
Universidades del Estado de Chile, 2015). Existe a su vez cierta desigualdad en la representación de hombres y mujeres en los puestos que componen el gobierno universitario de estas instituciones (Gaete-Quezada, 2015), lo que reafirma una cierta tendencia mundial que señala que mientras más altos son los cargos de responsabilidad en la institución, menor es la presencia de mujeres, pese a la inversión o incremento de participación de las mujeres en el sistema (Ordorika, 2015). La literatura especializada da cuenta de la presencia de las mujeres en las universidades en cargos y con énfasis en la ocupación de los directivos (Cuevas-López y Díaz-Rosas, 2015; Gaete-Quezada, 2015; Lorenzo et al., 2007; Montané y Pessoa de Carvalho, 2012; Negri, 2011; Peterson, 2016; Tomàs y Guillamón, 2009; Zuluaga y Moncayo, 2014).

\section{Mujeres en altos cargos directivos}

La feminización de la fuerza laboral demuestra que las mujeres alcanzan competencias y calificaciones necesarias para acceder a nuevos cargos de responsabilidad directiva (Matteo, 2012). Es así como la influencia de género en el mundo empresarial y la presencia de mujeres en altos cargos directivos ha sido estudiada especialmente en grandes empresas (Campbell y Mínguez-Vera, 2008). Se ha establecido que por el lado de la empresa, la promoción de la diversidad resulta ser un factor básico para innovar, crear elementos de ventaja competitiva y finalmente escalar y crecer en su trayectoria evolutiva (Pizarro y Guerra, 2010). Se cuenta con importantes investigaciones que relevan la irrupción de las mujeres empresarias en el desarrollo de las economías de todo el mundo (Bullough, Sully, Abdelzaher y Heim, 2015) y las motivaciones que las han llevado a ser empresarias (Rey-Martí, Tur Porcary Mas-Tur, 2015).

Al igual que a nivel internacional, la conformación de la gran empresa en Chile está marcada por una fuerte "subrepresentación" de la mujer en cargos gerenciales y de alta dirección, poniendo esto de manifiesto solo una de las dimensiones de la desigualdad de género presentes en el mercado laboral (Correll, Benard y Paik, 2007; Pizarro y Guerra, 2010). Al año 2014, la evidencia internacional indica que las mujeres en puestos de alta dirección llegan al 24\%, siendo Rusia el país donde existe un mayor número de mujeres en cargos gerenciales con un 43\% (ONU Mujeres, 2014).

Se han esgrimido que las limitaciones u obstáculos en el ascenso a los cargos de responsabilidad subrayan su origen predominantemente psicológico, social, económico, ideológico y cultural (Selva, 2012). Otra perspectiva y objeto de estudio para explicar la baja participación de las mujeres en la alta dirección son los descritos como las "aspiraciones", referidos a la falta de ambición por parte de mujeres (Cárdenas et al., 2010).En contraposición a lo anterior, se ha mencionado que el poder participar en esferas de alta responsabilidad responde a una necesidad de desarrollo, que a modo particular no debiera verse restringida por la condición sexual (Pizarro y Guerra, 2010).Y en términos de evolución personal, la mujer también ha experimentado el cambio a través 
de lo que se ha denominado la "ambición femenina", que supone que la mujer quiere asumir diversos roles de forma total en diversos órdenes de la vida (Selva, 2012).

Se ha enfatizado que la baja presencia de la mujer en cargos gerenciales tiene que ver con la propia organización, con la dinámica familiar, valores y prejuicios de la sociedad y las ideas preconcebidas sobre sí misma que obstaculizan su participación en los espacios de dirección. Sin embargo, las empresas no se ven como instituciones que siguen perpetuando relaciones desiguales entre hombres y mujeres (Ramos, Barberá y Sarrió, 2003). La inclusión de las mujeres en puestos de dirección y órganos de gobierno ha sido concebida como una manera de incrementar la diversidad de perspectivas y de opiniones en las empresas (Terjesen y Singh, 2008). Más aún, algunas investigaciones evidencian relaciones positivas entre las mujeres y los miembros de los directorios y el rendimiento de la empresa (Adler, 2001; Daily y Dalton, 2003; Krishnan y Park, 2005). Sin embargo, en Chile el estudio de Huerta, Contreras, Almodóvar y Navas (2015) estableció que el género del directivo no influye sobre los resultados de las empresas ya que no fue significativo desde el punto vista estadístico.

Según Cárdenas (2013), en su estudio en Chile aplicado a varias empresas en el año 2013 con 5.106 puestos de toma de decisión (presidencias, gerencias, direcciones superiores), tanto en el ámbito público como privado, evidenció que en todos los sectores se advierte una pirámide de poder y en la medida en que se asciende las mujeres ocupan porcentualmente menos cargos directivos, con una significativa disminución de la presencia de mujeres en altos cargos de dirección a nivel nacional, solo con un $21,7 \%$ de las mujeres (1.107 casos), mientras los hombres (3.999 casos) concentran el 78,8\% de los cargos estudiados. En tanto, los sectores donde se reflejan los porcentajes de mujeres en cargos de alta responsabilidad son el ámbito de la educación (27\%) y dirección de servicios públicos (34,1\%).

\section{Ascenso en las organizaciones y techos de cristal}

A partir de los años ochenta, el fenómeno que explica la barrera laboral invisible en la carrera de las mujeres (Tomàs \& Guillamón, 2009), y que explica desde la sociología, psicología y economía la discriminación en el ascenso de las mujeres en las organizaciones, se ha denominado "techo de cristal" (glass ceiling). Estos techos de cristal operan como una pared invisible, prácticamente infranqueable, construida por creencias, estereotipos, procedimientos, estructuras y relaciones de poder. Es así como se pueden encontrar distintas investigaciones, seminales y tendenciales, que han acuñado el concepto de los techos cristal, siendo ampliamente trabajado y definido en los últimos 30 años en publicaciones y que en general apuntan al conjunto de barreras invisibles que impide que las mujeres tengan iguales oportunidades que los hombres para el ascenso a los cargos de mayor jerarquía (Gaete-Quezada, 2015; Guil, 2014; Jackson y O'Callaghan, 2009; Tomàs y Guillamón, 2009; Zabludovsky Kuper, 2015). 
Otra forma de referirse a las barreras que frenan el desarrollo de las mujeres es el concepto de "muros de concreto", que engloba a los factores que impiden el avance en la vida política, en la educación y en los negocios. En la medida que van logrando tener acceso a más oportunidades y disfrutar de mayores derechos, las barreras ya no se manifiestan en leyes o normas escritas ni códigos visibles; se construyen como parte de una cultura empresarial caracterizada por una estructura jerárquica regida por reglas y prototipos masculinos y por condiciones organizacionales que pueden favorecer la discriminación, el acoso y las menores oportunidades para las mujeres (Cárdenas et al., 2010; Eagly y Carli, 2007).

Metafóricamente, Tomàs y Guillamón (2009) citan a Heward y a Estebaranz para referirse al fenómeno del "suelo pegajoso"(stickyfloor) desarrollado para ejemplificar que las mujeres caminan sobre un suelo, en la base de una pirámide, que las engancha y no les permite desprenderse para acceder a posiciones de nivel superior. Desde otra perspectiva, algunos autores afirman que las propias mujeres hacen suyo el mecanismo de exclusión, manteniendo un techo de cristal sobre sus cabezas, delimitando el desarrollo de sus carreras y estableciendo un tope en ellas (Santos Guerra citado por Tomàs y Guillamón, 2009).

Por su parte, Eagly y Carli (2007) proponen metafóricamente el concepto del "laberinto" con el propósito de describir los caminos alternativos y opciones de salida que utilizan las mujeres en su recorrido, que dejan de manifiesto los obstáculos que se ven enfrentados en el ascenso y que las llevan a renunciar en el camino, retrasando sus posibilidades de ascenso. Lo anterior se suma a los salarios inferiores y a la desigualdad en las condiciones laborales.

Complementariamente, a la preocupación básica por las desiguales condicioneslaborales entre mujeres y hombres, se ha sumado otra desigualdad, las llamadas "fronteras de cristal" (Burin, 2008), que se imponen a las mujeres cuando deben decidir entre familia y trabajo.

Respecto a la presencia de los techos de cristal en las universidades, foco de esta investigación, hay estudios importantes donde se evidencia que la presencia de mujeres es limitada en los cargos de alta dirección universitaria, independientemente del nivel de calificaciones y méritos que estas han alcanzado en los últimos años (Castro y Tomás, 2010; Jackson y O'Callaghan, 2009; Saracostti, 2006; Tomàs y Guillamón, 2009; Wylie, Jakobsen y Fosado, 2007), hecho que es replicable a la realidad actual de las universidades chilenas (Gaete-Quezada, 2015), dada por una representación desproporcionada de género, diferencias de compensación, rango y posición jerárquica. Entre las últimas investigaciones para el caso de las universidades públicas chilenas, Gaete-Quezada (2015) establece las dificultades y baja representación de las mujeres para acceder a los niveles directivos, con la presencia de techos de cristal en cinco universidades públicas. 


\section{Presencia de las mujeres en la gestión en las universidades}

La universidad como institución social se dedica a la transmisión, cultivo y creación de conocimiento, y aunque difiere de un país a otro, en su tamaño, en la cobertura de sus funciones, en el origen de propiedad u otros factores, está viviendo importantes transformaciones a nivel demográfico, económico, social, tecnológico y de competitividad nacional e internacional que han significado reformas en el gobierno de las instituciones que las componen. Pudiendo establecer que estos cambios también repercuten en los sistemas de dirección, organización y gestión de las instituciones de educación superior (Llinàs-Audet, Girotto, y Solé Parellada, 2011). Es así como las decisiones y acciones que las universidades implementan para cumplir sus objetivos se basan en las capacidades y habilidades de las personas que ocupan puestos de alta dirección, alineados con los procesos de dirección estratégica en los ámbitos de gestión académica, administrativa y económica.

El papel del administrador en los planteles de educación superior se reconoce como primordial para el liderazgo educativo, ya que estas instituciones tienen un rol fundamental en la estructura de la sociedad debido a su alto compromiso no solo en la formación de profesionales sino en la creación de conocimiento (Zuluaga y Moncayo, 2014). Siendo cada vez más explícito que no existen diferencias significativas entre hombres y mujeres líderes y sus necesidades de formación de gestión para dirigir este tipo de organizaciones (Sánchez-Moreno, López-Yañez y Altopiedi, 2015). En este ámbito, diagnósticos sobre la participación de la mujer en la educación superior reconocen que se ha dado ciertos avances en el mejoramiento de la situación de desigualdad de género, sin embargo, estos esfuerzos no han sido suficientes a pesar de la inversión educativa en las mujeres, persistiendo las dificultades para que las mujeres accedan a puestos estratégicos en la toma de decisiones y en los puestos de poder (Kiss et al., 2007).

Al respecto, Tomàs y Guillamón (2009) destacan las barreras y dificultades para acceder a cargos académicos en las universidades y establecen razones de tipología interna y externa. Entre las internas se citan los llamados procesos de socialización, entre los que se destacan: diferencias en la educación de niños y niñas, miedo a defraudar las expectativas del papel femenino, falta de modelos donde mirarse, poco interés por el estilo de liderazgo tradicional o una baja autoestima. A su vez, entre las razones externas se establecen: la cooptación, la dificultad de conciliación entre vida profesional y familiar, la cultura sexuada de las universidades y los estereotipos ligados al género femenino y al liderazgo.

El menor número de mujeres que participa en los órganos colegiados de los gobiernos de las universidades se acentúa aún más producto de la complejidad en la forma de gobernanza de las universidades (Ganga, Garrido, Abello y Vázquez, 2014) y desemboca en muchos casos en los conflictos de intereses al interior de la estructura 
organizacional en los distintos espacios de poder, siendo las universidades una organización no exenta de los conflictos de agencia.

Diversos estudios han abordado el tema de la gestión y del género en la educación superior, en un contexto donde se establece claramente una baja presencia de las mujeres en roles de docente e investigador en las universidades y más aún en cargos de gestión (ver Cuadro 1).

Se puede inferir cómo las universidades van más rezagadas en la ocupación de cargos de alta dirección por las mujeres que lo que sucede en las empresas públicas y privadas. Por ejemplo, se evidencia a nivel del sistema español que en 2009 solo cuatro de las setenta y tres universidades estaban dirigidas por una rectora, estableciendo claramente que el género persiste como criterio diferenciador de poder en las universidades (Tomàs y Guillamón, 2009). En este estudio, al revisar los porcentajes de docentes mujeres y hombres en el sistema español, se establece que por cada plaza una de cada tres es mujer, como a su vez persisten diferencias en la categorización y en especial en el bajo porcentaje en la cúpula académica.

Se ha señalado por algunos autores que la mujer cuando no masculiniza su poder conduce con un estilo más relacionado con lo informal, que en general descoloca a los hombres, pero que a su vez abre o permite nuevos caminos a otras mujeres (Negri, 2011). En las universidades se establecen ciertas premisas en un contexto abiertamente desigual e inequitativo, aunque en el discurso se plantea lo contrario. Siendo las universidades "instituciones meritocráticas" por excelencia, se establece que quien asciende más rápido es quien más sabe. Sin embargo, los hechos revelan otra situación (Kiss et al., 2007). En este sentido, Negri (2011) establece que las mujeres en las universidades tienen su techo de cristal en subsecretarías y vicedecanatos, acotando así sus espacios de poder. De esta forma, en el corto plazo, la presencia de mujeres en cargos de conducción universitaria no indicaría un cambio sustantivo que facilite el acceso de otras mujeres a los cargos de conducción, pero en largo plazo esto puede revertirse y, de hecho, se va dando cuando existen mujeres en cargos de dirección y gestión, lo que facilita el empoderamiento de otras mujeres.

Por otro lado, el acceso a cargos directivos está directamente relacionado a su vez con la trayectoria académica, situación de mérito que también es para los hombres. Algunas investigaciones han encontrado que la discriminación contra la mujer persiste en el mundo académico a pesar de los esfuerzos realizados. Uno de los aspectos de este problema es la promoción y tenencia de los cargos; varios estudios reportan que las profesoras son menos propensas a ser promovidas en comparación con sus colegas varones y la proporción de mujeres en la misma categoría disminuye a medida que aumenta de jerarquía (Lee y Won, 2014). 
Cuadro 1. Estudios de género en la gestión de universidades

\section{Evidencias}

Guil, Solano y Álvarez (2005). Percepción de las mujeres que trabajan en las universidades públicas andaluzas a partir de un enfoque subjetivista, combinando metodología cualitativa y cuantitativa. A partir de sus percepciones se determina factores de igualdad dentro del ámbito laboral.

Saracostti (2006). En un estudio de caso de cuatro universidades chilenas, investiga las posibilidades y tensiones en posiciones de poder por parte de las mujeres, abriendo un debate sobre poder y género.

Lorenzo, Sola y Cáceres (2007). La presencia de la mujer en los cargos directivos en un estudio descriptivo realizado en la Universidad de Granada, sobre el número de cargos de más alta representación institucional como decano, secretario y directores de departamentos.

Tomàs y Guillamón, (2009). Conocer los motivos de la baja presencia de las mujeres en roles de docente e investigador en las universidades y más aún en cargos de gestión e intenta determinar las barreras para las profesoras universitarias y se proponen algunas medidas correctivas.

Elizondo, Novo y Silvestre (2010). Análisis de la igualdad de mujeres y hombres en el sistema universitario español, determinando cuantitativa y cualitativa, a través de distintos estadísticos, el nivel de igualdad alcanzado por diferentes universidades públicas como privadas.

Negri (2011). Estudio de caso en universidad argentina utilizando el género como categoría de análisis, en un estudio de la composición de los órganos colegiados donde se explica las posibilidades de acceder a mejores posiciones en la jerarquía académica y la gestión.

Montané y Pessoa de Carvalho (2012). Análisis comparativo de las políticas de igualdad de oportunidades en Brasil y España, como las principales preocupaciones relacionadas con la presencia de mujeres y hombres en el contexto universitario, tanto del acceso, la distribución, promoción académica y producción de nuevas áreas de conocimiento.

Zuluaga y Moncayo (2014). Explora el acceso de mujeres a cargos de administración educativa y las características de liderazgo de mujeres académicas en cargos directivos en universidades de Bogotá, Colombia, Identificando los obstáculos internos/externos que reproducen la discriminación y un análisis en el liderazgo.

Gaete-Quezada (2015). Analiza el techo de cristal en el gobierno de las universidades estatales chilenas, tanto desde la participación como desde los obstáculos para acceder a ese tipo de puestos. Se concluye la existencia de desigualdades en la representación.

Matus-López y Gallego-Morón (2015). Presencia de los techos de cristal en un estudio de caso de una universidad española a partir de percepciones según perfiles. Los resultados son sometidos a test de contrastes no paramétricos.

Cuevas-López y Díaz-Rosas (2015). Descripción de la presencia de mujeres en dos de los ámbitos de gobierno más importantes de la universidad pública española: los rectorados, y los decanatos de facultades o las direcciones de escuelas técnicas superiores.

Sugur y Cangöz (2016). Investiga la subrepresentación de las mujeres en la gestión universitaria de Turquía, concluyendo que las mujeres académicas no tienen percepción de la "discriminación de género", pero sí enfrentan dificultades.

Remes y Cervera (2016). Investigación con la finalidad de visibilizar las relaciones asimétricas entre académicas y académicos en las Instituciones de Educación Superior de Guanajuato, México, hallándose poca presencia de mujeres en áreas de ingenierías y ciencias exactas y la existencia de brechas de cristal. 
Al comparar la situación universitaria de España y Brasil (Montané y Pessoa de Carvalho, 2012), se establece que la proporción de mujeres que imparten docencia se va reduciendo a medida que se sube de nivel jerárquico. Esto se explica porque las mujeres se concentran en carreras asociadas a roles tradicionales o bien en instituciones de menor prestigio social (Kiss et al., 2007), sumado a que el acceso masivo de las mujeres a la universidad es un hecho reciente. Este "techo de cristal" al que se enfrentan las mujeres es consecuencia de una cultura masculina que, por lo general, es la que predomina en el mundo académico. No obstante, este fenómeno es motivo de preocupación de algunos gobiernos y ha desencadenado lentamente acciones políticas para abordar la segregación vertical.

Tal como se señaló anteriormente, estudios recientes en Chile muestran que mientras más alto el nivel de la institución de educación, menor es la presencia de mujeres en cargos de responsabilidad superior. Esto es coincidente con los estudios de Wylie, Jakobsen y Fosado (2007) que establecen que en las universidades, aunque las mujeres acceden cada vez con mayor fuerza a más altos niveles de cualificación, siguen teniendo puestos de más baja jerarquía y contrataciones menos permanentes, situación coincidente con lo expuesto por Ordorika (2015) para México y otras latitudes. Así también, mientras más alto es el puesto de responsabilidad dentro de la institución, menor presencia de mujeres. Y destaca el caso que entre más alejada la institución de la capital, menor es la presencia de mujeres en cargos de responsabilidad superior (Cárdenas, 2013). En resumen, para el caso español, las causas de exclusión de las mujeres se encuentran en barreras externas, propias de la estructura social existente y que constituyen trabas para su desarrollo profesional (Tomàs y Guillamón, 2009).

\section{METODOLOGÍA}

El estudio se centra en las dieciséis universidades públicas chilenas existentes al año 2014. En una primera etapa se abocó a revisar en las distintas páginas web los miembros de la alta dirección, identificando tres niveles de cargo en concordancia con el estudio de Tomàs y Guillamón (2009). Estas autoras identificaron en el nivel uno: el cargo de rectorado y vicerrectorado; en el nivel dos: la delegación de rectorado, el decanato o la dirección de una escuela universitaria o un departamento; y en el nivel tres, los cargos de vicedecanato, jefatura de estudios, secretaría académica o coordinación/dirección de titulación, área, tercer ciclo, servicio o unidades. Se tomó en cuenta la propuesta de Castro y Tomás (2010) quienes clasifican a la estructura de dirección universitaria en unipersonales o colegiados y según su ámbito de influencia en cargos generales o periféricos. En el caso del estudio desarrollado, se innovó metodológicamente al revisar la constitución de los directorios o juntas directivas de las universidades para el caso chileno. 
Tal como se señaló, la información obtenida fue organizada en concordancia con la metodología de Tomàs y Guillamón (2009) y de Castro y Tomás (2010) para clasificar la estructura de dirección universitaria. Sin embargo, se pudo apreciar algunas diferencias que eran características de la estructura de las universidades públicas en Chile, pudiendo establecerse cinco niveles:

- Directorio o Junta Directiva: constituye el órgano superior que se conforma por un número limitado de directores, algunos designados por el gobierno de turno, los órganos académicos internos y otros representantes destacados de las actividades de la región. Posee importantes atribuciones de carácter estratégico en lo académico y económico.

- Rector: funcionario superior encargado de la dirección y supervisión de todas las actividades académicas, administrativas y financieras de la universidad. Dichos cargos son ocupados por académicos y se han sometido a algún tipo de sufragio, siendo ratificados por sus juntas directivas y por el presidente de la República.

- Equipo de rectorado: equipo de alta dirección universitaria que depende del rector de turno más el contralor. A este nivel es donde se encuentra la mayor heterogeneidad de cargos, dependiendo generalmente del tamaño, localización y nivel de descentralización, es así como podemos encontrar a lo menos cinco cargos que son más o menos comunes entre las universidades, como son: prorrector, vicerrector académico, vicerrector económico, contralor y secretario general. También es posible apreciar una cantidad de otras vicerrectorías, directores de sede o también direcciones generales con dependencia directa del rector.

- Decanos y vicedecanos: responsables de dirigir académica, administrativa y financieramente una facultad, estructura central que imparte docencia, investigación y otras actividades propias del quehacer universitario. En tanto, encontramos algunas universidades que poseen vicedecanos o secretarios de Facultad indistintamente, como aquella autoridad unipersonal que integra el equipo de gestión y coordina las políticas internas fijadas por el decano.

- Directores de departamento: responsable de dirigir académica, administrativa y financieramente un departamento, que es una unidad dependiente de una facultad que desarrolla una o más funciones básicas de la universidad en una disciplina perteneciente al área de conocimiento de esa facultad.

En la segunda etapa del estudio y con el objetivo de relacionar la presencia de las mujeres con factores de desempeño se utiliza un modelo logit, en un análisis análogo a los estudios de empresa y resultados financieros, adaptándolos al sistema universitario y estableciendo tres ámbitos de gestión posibles de medir: i) calidad académica medida a través de los años de acreditación institucional, ii) calidad investigativa con presencia en ranking latinoamericano QS y iii) desempeño financiero con tres índices. 
La información recopilada fue organizada en planilla Excel y trabajada con el paquete estadístico $R$. Se utilizó un análisis descriptivo para establecer una caracterización de la alta dirección. Posteriormente, se realizó un análisis que permitió establecer una relación causa-efecto entre las variables dependientes e independientes, con el fin de relacionar cantidad de mujeres en altos cargos universitarios y desempeño en la gestión a través de un modelo logit, definiendo las siguientes variables, que se detallan en la Tabla 1.

Tabla 1. Variables del modelo logit para desempeño de universidades

\begin{tabular}{|c|c|c|}
\hline Variables & Tipo & Descripción \\
\hline \multicolumn{3}{|l|}{ MUJERES } \\
\hline $\begin{array}{l}\text { Número de mujeres en } \\
\text { la alta dirección }\end{array}$ & Independiente & $\begin{array}{l}\text { Variable dummy: } 0 \text { y } 1 \text { para identificar la condición } \\
\text { de hombre y mujer. }\end{array}$ \\
\hline \multicolumn{3}{|c|}{ DESEMPEÑO UNIVERSIDAD EN ACREDITACIÓN } \\
\hline $\begin{array}{l}\text { Años acreditación } \\
\text { dicotómico }\end{array}$ & Dependiente & $\begin{array}{l}\text { 1: Con años de acreditación mayor al promedio (alto) } \\
0: \text { Con años de acreditación menor al promedio } \\
\text { (bajo) }\end{array}$ \\
\hline \multicolumn{3}{|c|}{ PRESENCIA EN RANKING QS LATINOAMERICANO } \\
\hline Ranking QS dicotómico & Dependiente & $\begin{array}{l}\text { 1: Universidad con presencia en ranking QS (alto) } \\
0: \text { Universidad sin presencia en ranking QS (bajo) }\end{array}$ \\
\hline \multicolumn{3}{|c|}{ DESEMPEÑO FINANCIERO DE LA UNIVERSIDAD } \\
\hline ROA dicotómico & Dependiente & $\begin{array}{l}\text { 1: Con ROA mayor al promedio sistema(alto) } \\
0: \text { Con ROA menor al promedio sistema(bajo) }\end{array}$ \\
\hline ROE dicotómico & Dependiente & $\begin{array}{l}\text { 1: Con ROE mayor al promedio sistema(alto) } \\
0 \text { : Con ROE menor al promedio sistema(bajo) }\end{array}$ \\
\hline Leverage dicotómico & Dependiente & $\begin{array}{l}\text { 1: Con leverage menor al promedio sistema(bueno) } \\
0: \text { Con leverage menor al promedio sistema(malo) }\end{array}$ \\
\hline
\end{tabular}

Fuente: Elaboración propia (2016)

Con el fin de obtener tres modelos se establecieron ciertos criterios metodológicos que se detallan a continuación.

Modelo 1: Desempeño por los años de acreditación institucional

La variable desempeño (rendimiento) fue operacionalizada en función de los años de acreditación que tiene cada casa de estudio a partir de los criterios emanados de la Comisión Nacional de Acreditación (CNA) de Chile. Para medir la diferencia entre un nivel alto o bajo de acreditación se establecerá el rango de años que ha establecido el órgano fiscalizador de uno a siete años y entendiendo, además, que una institución lo 
mínimo que debe acreditar son dos áreas obligatorias (gestión y docencia de pregrado). En este sentido, para valorar rendimiento se establecieron 2 parámetros:

1) Tener menos de 4 años de acreditación es un nivel inferior y 4 años o más un nivel alto de acreditación.

2) La cantidad de áreas que ha acreditado la casa de estudio. Realizando 3 áreas o más será considerado un nivel alto, e inferior a 3 será considerado como nivel bajo, ya que solo acredita lo mínimo.

Por consiguiente, al unir ambas variables se establece la condición de que sobre 4 años y con 3 áreas que entrega la universidad será considerado como alto, con variable dummy 1. A su vez una condición inferior o igual a 4 años y 2 áreas será considerado como un nivel bajo, con variable dummy 0.

\section{Modelo 2: Desempeño por presencia en Ranking Latinoamericano QS}

Este modelo de desempeño tiene la misma base del modelo anterior, pero agregando otra condicionante como es la participación o no en el QS University Ranking: Latin América a las dos anteriores que ya poseía como es la variable ranking; es decir, si está la casa de estudio o no en un ranking internacional de universidades, llamado "QS University Rankings: Latino América 2015" (este ranking trabaja con los datos 2014 coherente con los datos de esta investigación). Por lo tanto, el modelo quedó conformado por los siguientes parámetros:

1) Tener menos de 4 años de acreditación es un nivel inferior y 4 años o más un nivel alto de acreditación.

2) La cantidad de áreas que realiza la casa de estudio. Realizando3 áreas o más será considerado un nivel alto, e inferior a 3 como bajo.

3) Si está dentro del ranking QS o no la casa de estudio.

Por lo tanto, al unir ambas variables si la universidad tiene una acreditación institucional sobre 4 años con 3 áreas y si se encuentra dentro del ranking internacional se consideró como alto, con variable dummy 1, e inferior a 4 años y 2 áreas y no está dentro del ranking se consideró como nivel bajo, con variable dummy 0.

\section{Modelo 3: Desempeño por indicadores financieros}

Un tercer modelo que busca explicar el desempeño (rendimiento) fue establecido con variables financieras, a partir de los datos publicados por el Ministerio de Educación. Las 
variables explicativas serán iguales a los modelos usados anteriormente, considerando la totalidad de mujeres y hombres para cada casa de estudio.

Es así que se han elegido tres variables relevantes: ROA (rentabilidad sobre los activos), ROE (rentabilidad sobre el patrimonio) y leverage (relación deuda/patrimonio).

Por tanto, el modelo quedará conformado por los siguientes parámetros:

1) Si el ROA y el ROE de la universidad es igual o superior al promedio y el leverage es menor al promedio, se considerará bueno con una variable dummy 1.

2) Si el ROA y el ROE de la universidad es menor al promedio y el leverage es igual o mayor al promedio, se considerará bueno con una variable dummy 0 .

\section{LIMITACIONES DE LA INVESTIGACIÓN}

A partir de la distinción por nivel se realizó una cierta estandarización de los datos para hacerlos comparables entre sí y agruparlos por la condición de responsabilidad en la estructura jerárquica. A diferencia de los trabajos de Tomàs y Guillamón (2009) y Castro y Tomás (2010) se excluyó el nivel de Direcciones de Escuela, por la heterogeneidad de cargos, desactualización y falta de información en las distintas universidades. Otra información que se excluyó debido a su limitado acceso fue el nivel de gestión académica y administrativa a altura intermedia de la estructura organizativa, donde existe una alta heterogeneidad entre las instituciones. Caso especial en el tratamiento de los datos es la Universidad de Chile, ya que posee una estructura organizacional que difiere de las demás instituciones estatales al contar con un senado universitario, caso contrario se estarían duplicando los cargos. Es importante precisar que esta investigación limita su análisis de los techos de cristal a partir de datos cuantitativos disponibles en las universidades estatales chilenas, lo que puede diferir de un análisis exploratorio con consultas a los propios actores, pudiéndose encontrar factores causales desde una perspectiva sicosocial.

\section{RESULTADOS}

A partir del estudio de campo, que significó revisar la estructura organizacional de las dieciséis universidades estatales, se han podido establecer distintos niveles de resultados que responden análisis estadísticos de carácter descriptivo a nivel general, y detalle de cada una de las instituciones como un análisis causal con un modelo logit que permite establecer la incidencia de las mujeres en el desempeño de las instituciones. 


\section{Presencia en cargos directivos y techos de cristal}

Se pudo identificar 847 cargos en alta dirección universitaria, donde la mayor concentración se da a nivel de directores de departamentos que alcanzan 350 cargos con un $41,3 \%$ del total, lo siguen las decanaturas con 178 cargos, lo que representa un $19,2 \%$, el equipo rectorado con 163 cargos con un 19,2\%, los cargos de los directorios o juntas directivas que alcanzan a 140 representantes con un $16,5 \%$, y los cargos de rectores en la misma cuantía de las universidades (16) con un 1,9\% del total. Se podría suponer una correlación entre el número de cargos directivos y el tamaño de la universidad, es decir a mayor tamaño de una universidad, explicado por la cantidad de matrícula de estudiantes, mayor sería el número de cargos directivos que ésta debiera tener; para ello se realizó un análisis de correlación entre tamaño y cargos de las dieciséis universidades, obteniendo un coeficiente de 0,77 . Lo que indica una correlación directa entre dos variables.

\section{Presencia de las mujeres en cargos de alta dirección universitaria}

Uno de los hallazgos más interesante ha sido encontrar la presencia de mujeres en puestos directivos en relación con los 847 cargos de alta dirección existentes en las universidades, donde la presencia de la mujer alcanza a 206 cargos, representando un $24,3 \%$ del total. Al realizar un análisis por nivel jerárquico según se muestra en la Tabla 2, encontramos lo siguiente: en los directorios o juntas directivas las mujeres están presentes en 25 cargos, lo que representa un 17,9\% del total de cargos disponibles en el nivel (140). En los cargos de rector y tal como fue mencionado anteriormente no hay presencia de mujeres, representando un $0 \%$ del total de cargos disponibles (16). En el nivel de los equipos de rectorado la presencia de mujeres alcanza a 41 puestos con un $25,2 \%$ del total de cargos disponibles en el nivel (163). En las decanaturas (incluidos los secretarios de facultad y vicedecanos) las mujeres ocupan 49 cargos del total de cargos disponibles en el nivel (178) lo que representa un $27,5 \%$, siendo el nivel en términos relativos donde más se concentran las mujeres.

Tabla 2. Mujeres en cargos directivos en universidades públicas

\begin{tabular}{lccccc}
\hline Nivel & Mujeres & Hombres & $\begin{array}{c}\text { Total } \\
\text { cargos }\end{array}$ & $\begin{array}{c}\text { Mujer } \\
(\%)\end{array}$ & $\begin{array}{c}\text { Hombre } \\
(\%)\end{array}$ \\
\hline Directorio o Junta Directiva & 25 & 115 & 140 & 17,9 & 82,1 \\
\hline Rector & 0 & 16 & 16 & 0,0 & 100,0 \\
\hline Equipo de Rectorado & 41 & 122 & 163 & 25,2 & 74,8 \\
\hline Decanos y vicedecanos (secretarios) & 49 & 129 & 178 & 27,5 & 72,5 \\
\hline Directores de departamento & 91 & 259 & 350 & 26,0 & 74,0 \\
\hline Total & 206 & 641 & 847 & 24,3 & 75,7 \\
\hline
\end{tabular}

Fuente: Elaboración propia (2016) 
Por último, a nivel de Directores de Departamentos las mujeres alcanzan a 91 cargos con un $26 \%$ del total en el nivel (350). Estos datos dan cuenta que las mujeres están presentes en mayor porcentaje en los niveles de menos poder y disminuyendo al pasar al siguiente escalón jerárquico coherente con los trabajos de Gaete-Quezada (2015) y Tomàs y Guillamón (2009), por tanto se puede inferir en esta investigación la presencia de los "techos de cristal" a partir de las decanaturas.

Al revisarlos 206 cargos directivos ocupados por mujeres en cada universidad, se puede destacar que las universidades Metropolitana de Ciencias de la Educación $(34,9 \%)$, Magallanes $(34,3 \%)$ y Playa Ancha $(34,3 \%)$ son las instituciones, en términos de magnitud, que tienen mayor cantidad de mujeres en sus cargos directivos. En tanto, las universidades de Talca (15,3\%), Antofagasta $(19,0 \%)$ y la Serena $(19,3 \%)$ son las que presentan menor cantidad de mujeres en cargos directivos (ver Tabla 3).

Tabla 3. Mujeres en cargos directivos por universidad

\begin{tabular}{lccc}
\hline Institución & $\begin{array}{c}\text { Número de } \\
\text { cargos }\end{array}$ & $\begin{array}{c}\text { Mujeres en } \\
\text { cargos }\end{array}$ & $\begin{array}{c}\text { Mujeres en } \\
\text { cargos (\%) }\end{array}$ \\
\hline Universidad de Chile & 145 & 32 & 22,1 \\
\hline Universidad de Santiago De Chile & 57 & 13 & 22,8 \\
\hline Universidad de Valparaíso & 42 & 9 & 21,4 \\
\hline Universidad Arturo Prat & 27 & 6 & 22,2 \\
\hline Universidad del Bío-Bío & 60 & 16 & 26,7 \\
\hline Universidad de Talca & 33 & 5 & 15,2 \\
\hline Universidad de Tarapacá & 47 & 10 & 21,3 \\
\hline Universidad de La Frontera & 55 & 11 & 20,0 \\
\hline Universidad de Los Lagos & 45 & 10 & 22,2 \\
\hline Universidad de Playa Ancha & 70 & 24 & 34,3 \\
\hline Universidad de Antofagasta & 58 & 11 & 19,0 \\
\hline Universidad de La Serena & 46 & 9 & 19,6 \\
\hline Universidad Tecnológica Metropolitana & 50 & 16 & 32,0 \\
\hline Universidad de Atacama & 34 & 7 & 20,6 \\
\hline Universidad Metropolitana Cs. Educación & 43 & 15 & 34,9 \\
\hline Universidad de Magallanes & 35 & 12 & 34,3 \\
\hline Total & 847 & 206 & 24,3 \\
\hline
\end{tabular}

Fuente: Elaboración propia (2016)

Niveles jerárquicos y techos de cristal

Al revisar los distintos niveles en la estructura organizacional de las universidades, se puede evidenciar que en los cinco niveles jerárquicos donde la presencia de mujeres alcanza a 206 cargos las universidades presentan características distintas. 
Directorios o Juntas Directivas: Los cargos totales alcanzan a 140, de los que solo 25 cargos son ocupados por mujeres. Esto representa un $17,86 \%$ con respecto a un $82,14 \%$ de hombres, tal como se muestra anteriormente, distribuyéndose en 13 universidades de las 16, donde las Universidades de Valparaíso y de Los Lagos no cuentan con mujeres en sus juntas directivas.

Rectoría: La evidencia empírica demuestra que en las 16 universidades estudiadas durante este periodo no existe mujer en el cargo de rectoría. Esta afirmación coincide con la baja presencia de rectoras en otras latitudes (Mollo y Sol, 2015). Más aún, haciendo una revisión histórica a la información disponible en la web de las distintas casas de estudios se puede establecer que es un patrón común.

Equipos de Rectoría: Dentro de los equipos de rectoría, también denominados gobiernos universitarios o centrales, de un total de 163 puestos a nivel de las dieciséis universidades existen 41 que son ocupados por mujeres, siendo la Universidad de Magallanes donde la participación de las mujeres en la alta dirección es la más importante con un $50 \%$ de los puestos disponibles. En tanto, donde no existe participación de mujeres es en las Universidades de la Frontera y de Atacama. Al realizar un análisis más detenido al interior de los cargos de rectoría, de los 48 puestos de vicerrectores se puede establecer que solo16 son ocupados por mujeres, representando un33,33\% y se concentran solo en 10 universidades de las 16 . Destaca que es el ámbito académico donde hay más vicerrectoras, alcanzando 7 puestos.

Decanatura: donde se incluyen los vicedecanos o secretarios de facultad, de un total de 178 cargos en el país 49 son ocupados por mujeres, lo que representa un $27,5 \%$ de los puestos totales. Destaca a este nivel la Universidad Metropolitana de Ciencias de la Educación (UMCE) y la de Atacama con un $50 \%$ de los cargos, seguidas por las de la Frontera y de Magallanes con un $40 \%$ de los puestos disponibles. En tanto, las universidades en las que no existen mujeres en dichos cargos son las de Antofagasta y Arturo Prat.

Ahora bien, para realizar un análisis más exhaustivo que ayude a explicar este fenómeno a nivel de las decanaturas, se procedió a una clasificación por las áreas de conocimiento UNESCO impartidas por cada casa de estudio y específicamente por las Facultades, encontrando 49 mujeres que ocupan cargos directivos en decanaturas, que se concentran en áreas de Ciencias de Salud (27\%), Educación (21\%), Ciencias Sociales (13\%) y Humanidades (10\%) (ver Tabla 4).

Direcciones de Departamento: De un total de350 cargos relacionados a directores de departamento, dentro de las dieciséis universidades estatales, 91 cargos directivos los ocupan mujeres, representando un $26 \%$ del total. El total de 91 cargos se encuentran 
distribuidos en nueve áreas de conocimiento UNESCO, donde algunas de ellas ocupan 2 departamentos, llegando a 100, donde las áreas de Salud concentran la mayor cantidad de cargos (40\%), seguidas por las Ciencias Sociales (16\%) y Educación (13\%) (ver Tabla 4).

Tabla 4. Presencia \% de mujeres en cargos de decanatura y departamentos

\begin{tabular}{lcc}
\hline Área Conocimiento UNESCO & $\begin{array}{c}\text { Decanatura } \\
(\%)\end{array}$ & $\begin{array}{c}\text { Departamento } \\
(\%)\end{array}$ \\
\hline Salud & 27 & 40 \\
\hline Educación & 21 & 13 \\
\hline Ciencias Sociales & 13 & 16 \\
\hline Humanidades & 10 & 4 \\
\hline Arte y Arquitectura & 8 & 3 \\
\hline Ciencias Naturales y Matemáticas & 6 & 12 \\
\hline Derecho & 6 & 5 \\
\hline Tecnología & 4 & 6 \\
\hline Agropecuaria y Ciencias del Mar & 4 & 1 \\
\hline
\end{tabular}

Fuente: Elaboración propia (2016)

Al comparar el área donde se concentran las mujeres en cargos de decanatura y de departamento, se puede evidenciar un coeficiente de correlación entre ambas de 0,85 con un nivel de significancia de 0,01. Lo anterior permite suponer que la participación de las mujeres tiene una cierta similitud tanto en la estructura piramidal y áreas de conocimiento de las unidades académicas, donde las áreas de Matemáticas, Ingeniería y Tecnología son las de menor presencia, coincidente con estudios anteriores (Remes y Cervera, 2015), inclusive haciendo la distinción de "ciencias aplicadas" con mayor presencia masculina en los cargos directivos y en áreas disciplinarias de las "ciencias humanas" con mayor presencia femenina acorde a la investigación de Mollo y Sol (2015).

\section{Desempeño de las universidades y presencia de mujeres en la gestión}

Respecto a la incidencia de las mujeres en cargos directivos en las universidades se optó por desarrollar un modelo de análisis logit para poder determinar la incidencia que puede tener la presencia de mujeres en cargos directivos en el desempeño de las universidades estatales, determinado por la cantidad total de hombres y mujeres en ellas. Para ello, se 
trabaja con variables dummy: 0 y 1 para identificar la condición de hombre y mujer, respectivamente. Los ámbitos a analizar se realizaron a través de tres modelos, cuyos resultados se muestran en la Tabla 5:

- Presencia de mujeres en el desempeño por años de acreditación institucional

- Presencia de mujeres en el desempeño por Ranking Latinoamericano QS

- Presencia de mujeres en el desempeño por indicadores financieros

Tabla 5: Resultados modelo logit

\begin{tabular}{lccc}
\hline Coeficientes & $\begin{array}{c}\text { Modelo 1: } \\
\text { Desempeño } \\
\text { Acreditación }\end{array}$ & $\begin{array}{c}\text { Modelo 2: } \\
\text { Desempeño en } \\
\text { Ranking QS }\end{array}$ & $\begin{array}{c}\text { Modelo 3: } \\
\text { Desempeño } \\
\text { financiero }\end{array}$ \\
\hline Intercepto & $-3,3657$ & 0,52032 & $-1,56602$ \\
$(0,2518)$ & $(0,705)$ & $(0,4154)$ \\
\hline Mujeres & $-0,1995$ & $-0,26723$ & -0.35567 \\
& $(0,2445)$ & $(0,181)$ & $(0,1380)$ \\
\hline Hombre & 0,2011 & 0.05581 & 0.14260 \\
& $(0,0858)^{*}$ & $(0,372)$ & $(0,0919)^{*}$ \\
& & & \\
\hline
\end{tabular}

Fuente: Elaboración propia (2016)

Los resultados del Modelo 1 muestran que al existir más mujeres en cargos directivos disminuye el desempeño en 0,199 puntos a un nivel de significancia del $100 \%$. Sin embargo, puesto que el nivel de significancia es máximo, invalida el resultado anterior. Por ende, no existen pruebas estadísticas significativas que demuestren que al haber más mujeres el rendimiento de la casa de estudio disminuye o aumenta por el solo hecho de contar con mayor número de éstas. En tanto, la presencia de hombres mejora el desempeño en 0,20 , pero posee un nivel de significancia a un 0,1 ; aunque es baja es poco significativa.

En el caso del Modelo 2, los resultados arrojan que al contar con más mujeres disminuye el rendimiento en las universidades en 0,267 puntos, caso contrario que lo que sucede con los hombres. Sin embargo, estos datos no son estadísticamente significativos, lo que no le da validez a aquellas cifras, demostrando que no existe relación entre el nivel de rendimiento y la cantidad de mujeres.

En el tercer modelo que busca explicar el desempeño (rendimiento) con variables financieras se puede establecer que la presencia de mujeres disminuye el rendimiento en 0,35 puntos, en comparación a los hombres que lo aumentan en un 0,14 con una significancia de 0,1 (poco significativa). Sin embargo, este resultado no es estadísticamente significativo, lo que evidencia que no hay correlación entre el rendimiento de las universidades con la cantidad de hombres o mujeres en cargos directivos. 


\section{CONCLUSIONES}

El aporte de la mujer ha sido fundamental en las organizaciones lo que se evidencia en investigaciones de distintas perspectivas y Chile no es la excepción, aunque aún prevalecen ciertas culturas y conductas que impiden cambios más profundos en las relaciones de género. Aunque se observan avances importantes no hay que desconocer que en el sector universitario, que supone es la génesis de importantes transformaciones sociales, esto se encuentra más rezagado, tanto en experiencias internacionales como nacionales, como se muestra en las investigaciones de Tomàs y Guillamón (2009) en el caso español y Gaete-Quezada (2015) para el caso general chileno; coincidente con esta investigación, que pudo establecer que de 847 cargos de alta dirección en las universidades públicas chilenas 206 son ocupados por mujeres, lo que representa un $24,3 \%$ de todo el universo, es decir una baja presencia de mujeres en cargos de alta dirección universitaria.

Entre los hallazgos más relevantes se pudo establecer que en las dieciséis instituciones públicas existe una alta concentración de mujeres en cargos de decanatura y de departamento, y en especial en áreas disciplinarias denominadas "ciencias humanas" y con menor presencia en las "ciencias aplicadas", coincidente con las investigaciones de otras realidades latinoamericanas (Remes y Cervera, 2015; Mollo y Sol, 2015).

Se puede inferir que en ocho de ellas existen evidencias de la existencia de los techos de cristal, resultado mayor al de Gaete-Quezada (2015), y en las otras ocho universidades no se puede establecer con tanta claridad. Sin embargo, en la mayoría de las universidades en el nivel más alto de su estructura existe menos presencia de mujeres que en niveles de menos jerarquía, deduciendo de cierta forma la existencia de techos de cristal. Dichos techos de cristal se observan a partir del nivel de decanatura, desde ese nivel hacia arriba en la pirámide organizacional es menor la presencia de mujeres. $O$, dicho de otra forma, en los niveles de directores de departamentos y decanatura es donde llegan más mujeres en comparación a los niveles de equipos de rectoría, rectoría y directorios. En el nivel más alto de la estructura organizacional existe menos porcentaje de mujeres que en niveles de menor jerarquía, lo que manifiesta de cierta forma la existencia de techos de cristal. A pesar de las evidencias encontradas es importante precisar las limitaciones a un análisis puramente cuantitativo, que podría servir de base y dar lugar a nuevas investigaciones que puedan profundizar sobre las causas de los techos de cristal, realizando un trabajo exploratorio con los propios actores del sistema.

Otro hallazgo es que se pudo constatar que existe una correlación entre el tamaño de la institución, explicado por la cantidad de estudiantes matriculados, con el número de cargos de dirección con un coeficiente de correlación de 0,78 (sig. 0,01). Ahora bien, no hay que desconocer la dispersión entre las instituciones donde a pesar de esta 
relación, algunas tienen una sobredotación de cargos y otras presentan una menor cantidad en relación con su tamaño.

Al realizar el análisis de desempeño se pudo establecer que la presencia de mujeres disminuye el rendimiento en ciertos puntos en comparación con los hombres, tanto en el desempeño en años de acreditación, ranking internacional y variable financiera. Los resultados no son estadísticamente significativos, lo que evidencia que no hay una relación causal entre el rendimiento de las universidades con la cantidad de hombres o mujeres en cargos directivos, dado que el factor género en la alta dirección de las universidades estatales no incide en el desempeño.

\section{REFERENCIAS}

Adler, R. (2001). Women in the Executive Suite. Correlate to High Profits. Harvard Business Review, 79, 3-18.

Améstica, L., Gaete, H., y Llinàs-Audet, X. (2014). Segmentación y clasificación de las universidades en Chile: desventajas de inicio y efectos de las políticas públicas de financiamiento. Ingeniare. Revista Chilena de Ingeniería, 22(3), 384-397.

Barrientos, F., y A. Hess. (2016). La diversidad de género en los directorios chilenos (Working papers 6.2016). Recuperado de http://centrogobiernocorporativo.uc.cl/Ver-documento/265-La-diversidadde-genero-en-los-directorios-chilenos.html

Benvin, E., y Perticara, M. (2007). Análisis de los cambios en la participación laboral femenina en Chile. Revista de Análisis Económico, 22(1), 71-92.

Berríos, P. (2007). Análisis sobre los profesores universitarios y desafíos para la profesión académica en Chile. Revista Calidad de la Educación Superior, 26, 39-53.

Brega, C., Durán, G., y Sáez, B. (2015). Mujeres Trabajando. Una exploración al valor del trabjo y la calidad del empleo en Chile. Recuperado de http://www.fundacionsol.cl/wpcontent/uploads/2015/03/Estudio-Mujeres-Trabajando-2015.pdf

Bullough, A., Sully, M., Abdelzaher, D., y Heim, W. (2015). Developing women leaders through entrepreneurship education and training. Academy of Management Perspectives, 29(2), 250-270.

Burin, M. (2008). Las "fronteras de cristal" en la carrera laboral de las mujeres. Género, subjetividad y globalización. Anuario de Psicología, 39(1), 75-86.

Campbell, K., y Mínguez-Vera, A. (2008). Gender diversity in the boardroom and firm financial performance. Journal of Business Ethics, 83(3), 435-451.

Cárdenas, A. (2013). Mujeres y Poder. Participación en espacios de decisión. Recuperado de http://haymujeres.cl/wp-content/uploads/2016/05/PPT-G\%C3\%89NERO-Y-PODER-VF-5marzo.pdf

Cárdenas, M., Eagly, A., Heller, L., Jáuregui, K., Rivadeneira, W., y Salgado, E. (noviembre de 2010). Percepción de mujeres directivas en Latinoamérica sobre determinantes de su ascenso. Trabajo 
presentado en el Congreso Internacional "Las políticas de equidad de género en prospectiva: nuevos escenarios, actores y articulaciones". FLACSO, Buenos Aires, Argentina.

Castro, D., y Tomás, M. (2010). El gobierno y la gestión de la universidad: estudio de los órganos unipersonales. Estudios Sobre Educación, 19, 165-184.

Correll, S. J., Benard, S., y Paik, I. (2007). Getting a Job : Is There a Motherhood Penalty? American Journal of Sociology, 112(5), 1297-1339.

Consorcio de Universidades del Estado de Chile. (2015). Consorcio de las Universidades Estatales. Recuperado de http://www.uestatales.cl/cue/

Cuevas-López, M., y Díaz-Rosas, F. (2015). Gender and leadership in the Spanish university. A study on the gender gap in university management. Education Policy Analysis Archives, 23(196), 1-22.

Daily, C., y Dalton, D. (2003). Women in the boardroom: Business, A business imperative. Journal of Business Strategy, 24, 8-10.

Eagly, A. H., y Carli, L. L. (2007). Through the labyrinth: The truth about how women become leaders. Boston, MA: Harvard Business School Press.

Elizondo, A., Novo, A., y Silvestre, M. (2010). Igualdad de mujeres y hombres en las universidades españolas. Madrid, España: Instituto de la Mujer

Gaete-Quezada, R. (2015). El techo de cristal en las universidades estatales chilenas. Un ánálisis exploratorio. Revista Iberoamericana de Educación Superior, 6(17), 3-20.

Ganga, F., Garrido, O., Abello, Ju., y Vázquez, K. (2014). Intereses divergentes en las universidades de Chile: una indagación desde la teoría. Opción, 30(75), 15-34.

Guil, A. (2014). Techos de cristal blindado. INFAD Revista de Psicología, 6(1), 129-134.

Guil, A., Solano, A., y Álvarez, M. (2005). La situación de las mujeres en las universidades públicas andaluzas. Sevilla, España: Consejo Económico y Social de Andalucía.

Huerta, P., Contreras, S., Almodóvar, P., y Navas, J. (2015). Impacto del género sobre los resultados organizacionales de las empresas chilenas. Revista de Ciencias Sociales, 21(3), 373-385.

Jackson, J., y O'Callaghan, E. (2009). What do we know about glass ceiling effects? A taxonomy and critical review to inform higher education research. Research in Higher Education, 50, 460-482.

Kiss, D., Barrios, O., y Alvarez, J. (2007). Inequidad y diferencia. Mujeres y desarrollo académico. Revista Estudios Feministas, 15(1), 85-105.

Krishnan, H., y Park, D. (2005). A Few Good Women-on Top Management Teams. Journal of Business Research, 58(12), 1712-1720.

Larrieta I., Velasco, E., y Fernández, S. (2015). Las barreras en el acceso de las mujeres a los puestos de decisión: una revisión de la situación actual y de las principales aportaciones de la literatura. Revista de Dirección y Administración de Empresas, 22,1-23.

Lee, Y., y Won, D. (2014). Trailblazing women in academia: Representation of women in senior faculty and the gender gap in junior faculty's salaries in higher educational institutions. The Social Science Journal, 51(3), 331-340. 
Lorenzo, M., Sola, T., y Cáceres, M. (2007). El liderazgo femenino en los cargos directivos: un estudio longitudinal en la Universidad de Granada (1990-2005 ). Educación y Educadores, 10(2), 177-194.

Llinàs-Audet, X., Girotto, M., y Solé Parellada, F. (2011). La dirección estratégica universitaria y la eficacia de las herramientas de gestión: el caso de las universidades españolas. Revista de Educación, 355, 33-54.

Matteo, C. A. (2012). La mujer en el entorno gerencial venezolano: perfil, características y desempeño. Revista Venezolana de Estudios de La Mujer, 17(38), 39-66.

Matus-López, M., y Gallego-Morón, N. (2015). Techo de cristal en la Universidad. Si no lo veo no lo creo. Revista Complutense de Educación, 26(3), 611-626.

Meller, P., Valdés, G., y Lara, B. (2011). Female discrimination at the professional level in Chile. Interciencia, 36(11), 823-830.

Ministerio de Educación de Chile. (2014). Servicio de Información de Educación Superior (SIES). Recuperado de http://www.mifuturo.cl/index.php/academicos-einvestigadores

Mollo, G., y Sol, M. (2015). Mujeres Directivas en las Universidades Nacionales Argentinas. Revista Ciencias Administrativas FCE UNLP, 3(5), 39-51.

Montané, A., y Pessoa de Carvalho, M. (2012). Diálogo sobre género: justicia , equidad y políticas de igualdad en educación superior (Brasil y España). Revista Lusófona de Educação, 21, 97-120.

Negri, G. (2011). ¿Está cambiando la visión y el apoyo de las mujeres en el poder hacia las demás mujeres? El caso de la Universidad Nacional de Luján entre 2002 y 2010. La Aljaba Segunda Época, 15, 143-157.

Ordorika, I. (2015). Equidad de género en la Educación Superior. Revista de La Educación Superior, 44(174), 7-17.

ONU Mujeres. (2014). Las mujeres y la economía. Recuperado de http://beijing20.unwomen.org/es/infocus/economy

Peterson, H. (2016). Is managing academics "women's work"? Exploring the glass cliff inhigher education management. Educational Management Administration y Leadership, 44(1), 11-127.

Pizarro, O., y Guerra, M. (2010). Rol de la mujer en la gran empresa. Un estudio del programa Chile Emprendedoras. Recuperado de http://www.udd.cl/wp-content/uploads/2009/11/Rol-dela-mujeren-la-gran-empresa-FINAL2010.pdf

Ramos, A., Barberá, E., y Sarrió, M. (2003). Mujeres directivas, espacio de poder y relaciones de género. Anuario de Psicología, 34(2), 267-278.

Remes, M., y Cervera, C. (2016). El Techo de Cristal en las universidades. Jóvenes en la ciencia, 2(1), 1767-1771.

Rey-Martí, A., Tur Porcar, A., y Mas-Tur, A. (2015). Linking female entrepreneurs' motivation to business survival. Journal of Business Research, 68(4), 810-814.

Sánchez-Moreno, M., López-Yañez, J., y Altopiedi, M. (2015). Leadership characteristics and training needs of women and men in charge of Spanish universities. Gender in Management, 27(3), 255272. 
Saracostti, M. (2006). Mujeres en alta dirección de educación superior: posibilidades, tensiones y nuevas interrogantes. Revista de Calidad en Educación, 25, 243-259.

Selva, C. (2012). Mujer y trayectoria profesional. ¿Un acceso igualitario a los cargos de dirección?. RIDEG, 2, 33-39.

Sugur, S., y Cangöz, L. (2016). Women and gender relations in university management. Amme Idaresi Dergisi, 49(3), 89-115.

Terjesen, S., y Singh, V. (2008). Female presence on Corporate Boards: A multi-country study of environmental context. Journal of Business Ethics, 83, 55-63.

Tomàs, M., y Guillamón, C. (2009). Las barreras y los obstáculos en el acceso de las profesoras universitarias a los cargos de gestión académica. Revista de Educación, 350, 253-275.

Wylie, A., Jakobsen, J. R., y Fosado, G. (2007). Women, work, and the academy. Nueva York, NY: The Barnard Center for Research on Women.

Zabludovsky Kuper, G. (2015). Las mujeres en los ámbitos de poder económico y político de México. Revista Mexicana de Ciencias Políticas y Sociales, 60(223), 61-94.

Zuluaga, D., y Moncayo, B. (2014). Perspectivas del liderazgo educativo: Mujeres académicas en la administración. Suma de Negocios, 5(11), 86-95. 